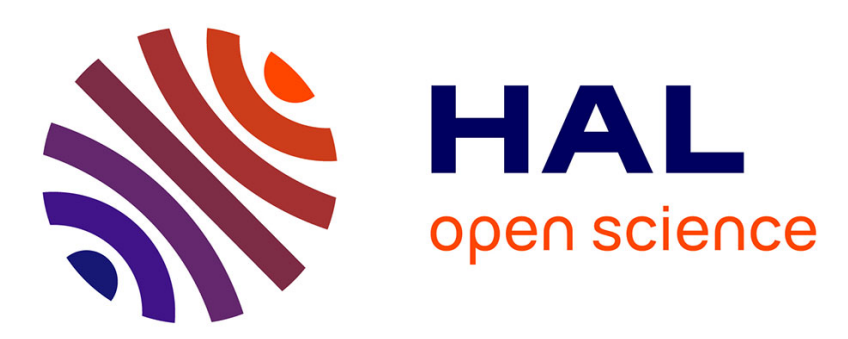

\title{
A two-impulse method for stabilizing the spacecraft relative motion with respect to a periodic trajectory
}

Georgia Deaconu, Christophe Louembet, Alain Théron

\section{To cite this version:}

Georgia Deaconu, Christophe Louembet, Alain Théron. A two-impulse method for stabilizing the spacecraft relative motion with respect to a periodic trajectory. 51st Annual Conference on Decision and Control (CDC 2012), Dec 2012, Maui, United States. 10.1109/CDC.2012.6426542 . hal01872905

\section{HAL Id: hal-01872905 \\ https://hal.laas.fr/hal-01872905}

Submitted on 12 Sep 2018

HAL is a multi-disciplinary open access archive for the deposit and dissemination of scientific research documents, whether they are published or not. The documents may come from teaching and research institutions in France or abroad, or from public or private research centers.
L'archive ouverte pluridisciplinaire HAL, est destinée au dépôt et à la diffusion de documents scientifiques de niveau recherche, publiés ou non, émanant des établissements d'enseignement et de recherche français ou étrangers, des laboratoires publics ou privés. 


\title{
A two-impulse method for stabilizing the spacecraft relative motion with respect to a periodic trajectory*
}

\author{
Georgia Deaconu $^{1,2}$, Christophe Louembet ${ }^{1,2}$ and Alain Théron ${ }^{1,2}$
}

\begin{abstract}
The article presents an analytical method for computing a two-impulse control law that stabilizes the spacecraft relative motion with respect to an invariant set. The invariant set contains the states belonging to a desired periodic relative trajectory and is described using linear equations. The two impulses are computed analytically based on the prediction of the evolution of the relative trajectory. Closed-loop tests are conducted using the non-linear relative dynamics, for different eccentricities of the reference orbit and for different levels of navigation uncertainty. Encouraging results are obtained using a control strategy that requires very few computational effort.
\end{abstract}

\section{INTRODUCTION}

The spacecraft ability to maintain a relative periodic motion will be an important aspect of future on-orbit inspection and on-orbit servicing missions [1]. Moreover, enabling the spacecraft to evolve on naturally periodic relative trajectories could greatly reduce the mission's fuel cost.

The periodic relative orbits are usually described using the closed-form solutions of the equations of relative motion [2], [3]. In a real environment, the spacecraft ability not to drift apart from a desired periodic trajectory is highly dependent on the accuracy of the model used for deriving these solutions. However, orbital disturbances such as gravitational perturbations and atmospheric drag, modelling errors from linearised dynamics or measurement noise will cause the spacecraft to move away from the periodic trajectory, unless a control action provides the necessary corrections.

The control strategies proposed in the literature for maintaining the spacecraft on periodic relative trajectories span over a large range of control techniques, including LQR, non-linear, Lyapunov, model predictive control, etc., and use either continuous or impulsive thrusting (see [4] and the references therein). The main challenges consist in finding an accurate but simple model of relative motion, valid for arbitrary elliptical reference orbits, and determining a stabilizing control law that guarantees an efficient trade-off between the performances, the complexity of the algorithm and the limited computational power available on-board [5].

Several recent publications address these challenges. [6] uses a time-delayed feedback control to stabilize and bound the spacecraft relative motion. Since only the period of the resulting trajectory needs to be specified, the method is not sensitive to modelling errors and can be applied to systems without a priori knowledge of their dynamics. However it does not provide any information about the shape or the

\footnotetext{
*This work was supported by CNES ans Astrium EADS

${ }^{1}$ CNRS ; LAAS ; 7 Avenue du colonel Roche, F-31077 Toulouse; France

${ }^{2}$ Univ de Toulouse; UPS, INSA, ISAE; UT1, UTM, LAAS; F-31077 Toulouse; France
}

dimensions of the obtained relative trajectory and it does not consider navigation uncertainty.

[7] presents a feedback controller based on the solution of a differential Riccati equation, that steers the spacecraft to a given periodic trajectory. The reference state is obtained by introducing a virtual satellite whose motion is propagated by integrating the complex non-linear dynamics, starting from initial conditions that ensure the periodicity of the trajectory. While effective from a modelling precision point of view, this method for retrieving the desired state can be computationally expensive and can suffer from loss of numerical accuracy when long propagation times are required.

To guarantee a priori robustness of the control law to navigation uncertainties, [8] turns towards more sophisticated control techniques. Model predictive control (MPC) is used for designing a fuel optimal manoeuvre plan leading from an initial state to the desired state. Moreover, a range of initial states is considered in the synthesis in order to capture the measurement uncertainty and to ensure robust performances. But the robust performances of the method come at the price of having to solve at each step a linear optimization problem.

The control law presented in this article is based on the linearised model for the spacecraft relative dynamics expressed in a local Cartesian frame. It consists of a twoimpulse manoeuvre that stabilizes the relative motion with respect to an invariant set, by requiring the distance to this set to become zero. The invariant set is composed of the states belonging to a specified periodic trajectory and it can be described using only linear equations. This enables the computation of the reference state at any moment by a simple matrix multiplication. The resulting control law is suitable for any kind of eccentric orbit and has the advantage of requiring very few computational effort. Its robustness properties to navigation uncertainties are shown through numerical simulations. However, the optimality in terms of fuel consumption is not guaranteed.

The article is organized as follows. Section II gives a brief description of the parametrization of a periodic spacecraft relative trajectory and analyzes the stability of the relative motion with respect to this invariant set. Section III presents the stabilizing two-impulse control method while section IV provides extensive numerical analysis to evaluate its performances.

\section{CHARACTERIZATION OF THE PERIODIC MOTION}

\section{A. Parametrization of the relative periodic trajectory}

Let us consider the relative motion between two spacecraft on elliptic Keplerian orbits, a passive leader and a controlled 
follower. The relative state $X \in \mathbb{R}^{6}$ consists of the relative position and the relative velocity between the spacecraft, expressed in the leader's local-vertical/local-horizon (LVLH) frame $X=\left[\begin{array}{llllll}x & y & z & v_{x} & v_{y} & v_{z}\end{array}\right]^{T}$.

The spacecraft relative motion under impulsive control $\Delta \tilde{V}$, is described using the Yamanaka-Akersen transition matrix [9] $\Phi_{v_{k}}^{v_{k+1}}$, solution of the Tschauner-Hempel equations [10] for the relative dynamics:

$$
\tilde{X}\left(v_{k+1}\right)=\Phi_{v_{k}}^{v_{k+1}}\left(\tilde{X}\left(v_{k}\right)+B \Delta \tilde{V}_{k}\right)=\Phi_{v_{k}}^{v_{k+1}} \tilde{X}^{+}\left(v_{k}\right)
$$

The matrix $B$ expresses the fact that the impulsive control acts only on the relative velocity. The independent variable is the true anomaly of the leader $v$ and the following variable change allows the passage from the time domain to the scaled variables in $v$ :

$$
\tilde{X}(v)=\left[\begin{array}{cc}
(1+e \cos v) I_{3} & 0_{3} \\
\frac{1+e \cos v}{\dot{v}} I_{3} & -e \sin v I_{3}
\end{array}\right] X(t)
$$

where $e$ is the eccentricity of the leader's orbit, $I_{3} \in \mathbb{R}^{3 \times 3}$ is the identity matrix and $0_{3} \in \mathbb{R}^{3 \times 3}$ is the zero matrix.

Searching for an autonomous periodic trajectory for system (1) is equivalent to imposing the passage through the same state $\tilde{X}\left(v_{k}\right)$ after one period of free motion:

$$
\tilde{X}\left(v_{k}+2 \pi\right)=\Phi_{v_{k}}^{v_{k}+2 \pi} \tilde{X}\left(v_{k}\right)=\tilde{X}\left(v_{k}\right)
$$

It follows a linear condition that the initial state of the propagation must satisfy in order for the trajectory to be periodic:

$$
M\left(v_{k}\right) \tilde{X}\left(v_{k}\right)=0
$$

where $M(v) \in \mathbb{R}^{1 \times 6}$ is defined by:

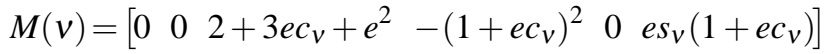

with $c_{v}=\cos v$ and $s_{v}=\sin v$.

By considering the autonomous motion in (1) and assuming that the initial state $\tilde{X}\left(v_{k}\right)$ satisfies (4), the parametric equations for the propagation of the periodic motion are obtained:

$$
\begin{aligned}
& \tilde{x}(v)=(2+e \cos v)\left(d_{1} \sin v-d_{2} \cos v\right)+d_{3} \\
& \tilde{y}(v)=d_{4} \cos v+d_{5} \sin v \\
& , v \geq v_{k} \\
& \tilde{z}(v)=(1+e \cos v)\left(d_{1} \cos v+d_{2} \sin v\right)
\end{aligned}
$$

The parameters $d_{i}, i=1 \ldots 5$ can be expressed as functions of the initial state of the periodic motion $\tilde{X}\left(v_{k}\right)$. Let $D=$ $\left[\begin{array}{lllll}d_{1} & d_{2} & d_{3} & d_{4} & d_{5}\end{array}\right]^{T}$, then:

$$
D=C\left(v_{k}\right) \tilde{X}\left(v_{k}\right)
$$

where the matrix $C(v) \in \mathbb{R}^{5 \times 6}$ is defined by:

$$
C(v)=\left[\begin{array}{cccccc}
0 & 0 & \frac{2 e c_{v}^{2}+c_{v}-e}{\left(1+e c_{v}\right)^{2}} & 0 & 0 & -\frac{s_{v}}{1+e c_{v}} \\
0 & 0 & \frac{s_{v}\left(1+2 e c_{v}\right)}{\left(1+e c_{v}\right)^{2}} & 0 & 0 & \frac{c_{v}}{1+e c_{v}} \\
1 & 0 & \frac{e s_{v}\left(2+e c_{v}\right)}{\left(1+e c_{v}\right)^{2}} & 0 & 0 & \frac{2+e c_{v}}{1+e c_{v}} \\
0 & c_{v} & 0 & 0 & -s_{v} & 0 \\
0 & s_{v} & 0 & 0 & c_{v} & 0
\end{array}\right]
$$

Equations (6) show that any two states $\tilde{X}\left(v_{k}\right)$ and $\tilde{X}\left(v_{j}\right)$ belonging to the same periodic trajectory respect the periodicity condition (4) and generate the same vector of parameters $D$ :

$$
C\left(v_{k}\right) \tilde{X}\left(v_{k}\right)=C\left(v_{j}\right) \tilde{X}\left(v_{j}\right)=D, \text { with } v_{k} \neq v_{j}
$$

This comes from the fact that when using (6) to propagate over one orbital period the motion starting from $\tilde{X}\left(v_{k}\right)$ and from $\tilde{X}\left(v_{j}\right)$, the obtained trajectories must coincide since we made the hypothesis that the two states belong to the same periodic trajectory.

This observation enables the computation of the reference state on a specified periodic trajectory at any given moment. A state $\tilde{X}\left(v_{k}\right)$ belongs to a periodic trajectory specified by the parameters $D$ if and only if:

$$
\left[\begin{array}{l}
M\left(v_{k}\right) \\
C\left(v_{k}\right)
\end{array}\right] \tilde{X}\left(v_{k}\right)=\left[\begin{array}{l}
0 \\
D
\end{array}\right]
$$

The corresponding state on the desired trajectory $D$ is deduced by inverting (10):

$$
\tilde{X}\left(v_{k}\right)=\left[\begin{array}{l}
M\left(v_{k}\right) \\
C\left(v_{k}\right)
\end{array}\right]^{-1}\left[\begin{array}{l}
0 \\
D
\end{array}\right]=Y\left(v_{k}\right)\left[\begin{array}{l}
0 \\
D
\end{array}\right]
$$

The matrix in (11) is always invertible since its determinant equals -1 and the matrix $Y \in \mathbb{R}^{6 \times 6}$ is defined by:

$$
Y(v)=\left[\begin{array}{cccccc}
0 & s_{v}\left(2+e c_{v}\right) & -c_{v}\left(2+e c_{v}\right) & 1 & 0 & 0 \\
0 & 0 & 0 & 0 & c_{v} & s_{v} \\
0 & c_{v}\left(1+e c_{v}\right) & s_{v}\left(1+e c_{v}\right) & 0 & 0 & 0 \\
\frac{-1}{\left(1+e c_{v}\right)^{2}} & 2 e c_{v}^{2}+2 c_{v}-e & 2 s_{v}\left(1+e c_{v}\right) & 0 & 0 & 0 \\
0 & 0 & 0 & 0 & -s_{v} & c_{v} \\
0 & -s_{v}\left(1+2 e c_{v}\right) & 2 e c_{v}^{2}+c_{v}-e & 0 & 0 & 0
\end{array}\right]
$$

\section{B. Stability around a periodic trajectory}

The stability around a periodic trajectory is understood in the sense given in [11], which requires the characterisation of the evolution of the trajectories that start arbitrarily close to the periodic one. An "asymptotically stable" behaviour of these neighbouring trajectories would imply their natural convergence towards the periodic solution. The stability properties of the periodic solutions are analysed by extending the notion of Lyapunov stability of an equilibrium point to the notion of stability of an invariant set [11].

A set $L \in \mathbb{R}^{n}$ is said to be positively invariant for a discrete autonomous system of the form:

$$
x\left(t_{k+1}\right)=f\left(x\left(t_{k}\right)\right)
$$

if, for all $x\left(t_{0}\right) \in L$, the solution $x\left(t_{k}\right) \in L$ for $t_{k} \geq t_{0}$. Hence the set $L$ is invariant if all the trajectories starting in the set remain in the set.

An invariant set $L$ is said to be stable if, for each $\varepsilon \geq 0$, it exists $\delta \geq 0$ such that :

$$
\forall x\left(t_{0}\right) \text { s.t. } \operatorname{dist}\left(x\left(t_{0}\right), L\right) \leq \delta \Rightarrow \operatorname{dist}\left(x\left(t_{k}\right), L\right) \leq \varepsilon, \forall t_{k} \geq t_{0}
$$


where the distance from a point $x$ to the set $L$ is defined by:

$$
\operatorname{dist}(x, L)=\inf _{y \in L}\|x-y\|
$$

An invariant set $L$ is said to be asymptotically stable if it is stable and $\delta$ can be chosen such that:

$$
\forall x\left(t_{0}\right) \text { s.t. } \operatorname{dist}\left(x\left(t_{0}\right), L\right) \leq \delta \Rightarrow \lim _{k \rightarrow \infty} \operatorname{dist}\left(x\left(t_{k}\right), L\right)=0
$$

This means that trajectories starting arbitrarily close to the set will converge towards the set.

Considering the autonomous evolution of (1):

$$
\tilde{X}\left(v_{k+1}\right)=\Phi_{v_{k}}^{v_{k+1}} \tilde{X}\left(v_{k}\right)
$$

and defining $S$ as the set composed of the states of system (17) belonging to all the possible periodic trajectories, regardless of their shape or dimensions:

$$
S=\left\{\tilde{X}(v) \in \mathbb{R}^{n} \mid M(v) \tilde{X}(v)=0\right\}
$$

it can be showed that $S$ is a stable invariant set.

Proposition 1: $\mathrm{S}$ is a stable invariant set for system (17). Proof: It can easily be checked that the state transition matrix $\Phi$ verifies the following property:

$$
M\left(v_{j}\right) \Phi_{v_{k}}^{v_{j}}=M\left(v_{k}\right) .
$$

Thus, if a state $\tilde{X}\left(v_{k}\right)$ respects the periodicity condition (4), then any following state $\tilde{X}\left(v_{j}\right)$, with $v_{j} \geq v_{k}$, will also respect the periodicity condition. Hence, $S$ is a positive invariant set for the autonomous system (17).

For any $\tilde{X}\left(v_{k}\right)$ such that

$$
\operatorname{dist}\left(\tilde{X}\left(v_{k}\right), S\right)=\left\|M\left(v_{k}\right) \tilde{X}\left(v_{k}\right)\right\| \leq \delta
$$

and for any $v_{j} \geq v_{k}$, we can write

$$
\operatorname{dist}\left(\tilde{X}\left(v_{j}\right), S\right)=\left\|M\left(v_{j}\right) \Phi_{v_{k}}^{v_{j}} \tilde{X}\left(v_{k}\right)\right\|=\left\|M\left(v_{k}\right) \tilde{X}\left(v_{k}\right)\right\| \leq \delta .
$$

It follows that the invariant set $S$ is stable since it satisfies (14), but not asymptotically stable since (16) is not verified, in the absence of a control action.

Remark 1: The stability property of $S$ does not guarantee a periodic autonomous evolution of the system. A trajectory that verifies (20) is a drifting trajectory, with the amount of drift per orbit fixed by $\delta$ (Figure 1). A control action needs to be applied in order to ensure the asymptotic stability leading to a periodic motion.

\section{CONTROL LAWS}

\section{A. Single-impulse control}

A simple way of computing a single-impulse manoeuvre $\Delta \tilde{V}=\left[\begin{array}{lll}\Delta \tilde{V}_{x} & \Delta \tilde{V}_{y} & \Delta \tilde{V}_{z}\end{array}\right]^{T}$ that brings the system on a periodic trajectory can be derived from the definition of the invariant set $S$. Based on the measurement of the current state $\tilde{X}\left(v_{k}\right)$, an impulsive control can be computed such that the state after control $\tilde{X}^{+}\left(v_{k}\right)$ verifies:

$$
\operatorname{dist}\left(\tilde{X}^{+}\left(v_{k}\right), S\right)=\left\|M\left(v_{k}\right)\left(\tilde{X}\left(v_{k}\right)+B \Delta \tilde{V}\right)\right\|=0
$$

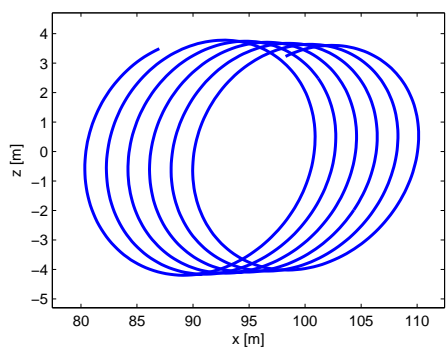

Fig. 1. Trajectory which satisfies (20), $\delta=0.1$

It follows that the single-impulse control must satisfy:

$$
-\Delta \tilde{V}_{x}+\frac{e \sin v_{k}}{1+e \cos v_{k}} \Delta \tilde{V}_{z}=-\frac{M\left(v_{k}\right)}{\left(1+e \cos v_{k}\right)^{2}} \tilde{X}\left(v_{k}\right)
$$

with the simplified version in case the designer makes the choice to apply control only on the $x$ axis:

$$
\Delta \tilde{V}_{x}=\frac{M\left(v_{k}\right)}{\left(1+e \cos v_{k}\right)^{2}} \tilde{X}\left(v_{k}\right)
$$

The impulsive control (23) or (24) will attempt to counteract the effect of the various orbital disturbances on the periodicity of the motion. It will drive the system towards a neighbouring periodic trajectory but it runs the risk of a resulting relative trajectory that is unbounded since no other restrictions are added. Figure 2 shows the evolution of the trajectory under $J_{2}$ perturbation when the impulsive control (24) is applied in closed-loop.

One way to fix this problem is to try to steer the system towards the closest periodic trajectory that is guaranteed to evolve inside a specified region. This could be done using model predictive control algorithms [4], [12] for instance, but at the price of solving an optimization problem on-line. Since analytical methods require fewer computational effort, they are more suited for on-board implementation. An analytical method for computing the control that steers the spacecraft to a predefined periodic trajectory is introduced next.

\section{B. Two-impulse control}

Let $D$ be a vector of parameters that defines a periodic trajectory as in (7). The invariant set composed of the states belonging to this trajectory is defined by:

$$
S_{D}=\left\{\tilde{X}(v) \in \mathbb{R}^{n} \mid M(v) \tilde{X}(v)=0, C(v) \tilde{X}(v)=D\right\}
$$

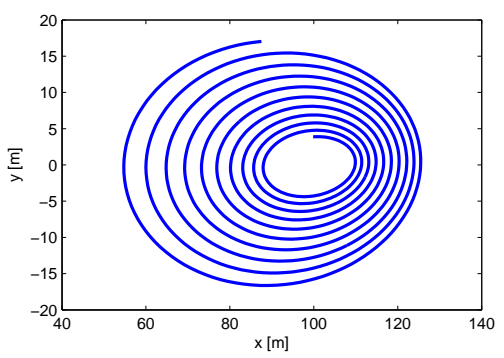

Fig. 2. Trajectory under single-impulse control and $J_{2}$ perturbation 
with the associated distance:

$$
\operatorname{dist}\left(\tilde{X}(v), S_{D}\right)=\left\|\left[\begin{array}{c}
M(v) \\
C(v)
\end{array}\right] \tilde{X}(v)-\left[\begin{array}{l}
0 \\
D
\end{array}\right]\right\|
$$

The purpose is to find a control law that stabilizes the system (1) around the periodic trajectory $D$, i.e. a control law that aims at obtaining:

$$
\operatorname{dist}\left(\tilde{X}(v), S_{D}\right)=0
$$

A two-impulse method provides an analytical solution to this problem. Starting from the current measurement $\tilde{X}\left(v_{k}\right)$, two controls $\Delta \tilde{V}_{k}$ and $\Delta \tilde{V}_{k+1}$ are computed, such that the state after the second thrust belongs to the desired periodic trajectory: $\operatorname{dist}\left(\tilde{X}^{+}\left(v_{k+1}\right), S_{D}\right)=0$. This leads to:

$$
\begin{gathered}
{\left[\begin{array}{c}
M\left(v_{k+1}\right) \\
C\left(v_{k+1}\right)
\end{array}\right]\left(\tilde{X}\left(v_{k+1}\right)+B \Delta \tilde{V}_{k+1}\right)=\left[\begin{array}{c}
0 \\
D
\end{array}\right]} \\
{\left[\begin{array}{c}
M\left(v_{k+1}\right) \\
C\left(v_{k+1}\right)
\end{array}\right]\left(\Phi_{v_{k}}^{v_{k+1}}\left(\tilde{X}\left(v_{k}\right)+B \Delta \tilde{V}_{k}\right)+B \Delta \tilde{V}_{k+1}\right)=\left[\begin{array}{c}
0 \\
D
\end{array}\right]}
\end{gathered}
$$

The following solution is obtained:

$$
\left[\begin{array}{c}
\Delta \tilde{V}_{k} \\
\Delta \tilde{V}_{k+1}
\end{array}\right]=\left[\begin{array}{ll}
\Phi_{v_{k}}^{v_{k+1}} B & B
\end{array}\right]^{-1}\left(Y\left(v_{k+1}\right)\left[\begin{array}{c}
0 \\
D
\end{array}\right]-\Phi_{v_{k}}^{v_{k+1}} \tilde{X}\left(v_{k}\right)\right)
$$

For the matrix to be invertible care must be taken to have $v_{k+1}-v_{k} \neq n \pi$. The control depends on the reference trajectory (through the vector $D$ ), on the current instant $v_{k}$ and on the interval between $v_{k}$ and $v_{k+1}$. It is computed based on the prediction of the evolution of the trajectory at $v_{k+1}$ since the terms in the parenthesis correspond to the reference state and to the autonomously propagated state respectively.

\section{Robustness to navigation uncertainty}

Suppose the measured state $\tilde{X}_{m}\left(v_{k}\right)$ lies in an uncertainty ellipsoid around the real state $\tilde{X}_{r}\left(v_{k}\right)$, ellipsoid defined by the matrix $P_{u} \in \mathbb{R}^{6 \times 6} \succeq 0$ :

$$
\tilde{X}_{m}\left(v_{k}\right)=\tilde{X}_{r}\left(v_{k}\right)+P_{u} u, u^{T} u \leq 1
$$

The control law (30) is computed starting from the measured state $\tilde{X}_{m}\left(v_{k}\right)$ but it is applied to the real state $\tilde{X}_{r}\left(v_{k}\right)$. This causes the final distance to the invariant set $S_{D}$ to be nonzero:

$$
\operatorname{dist}\left(\tilde{X}^{+}\left(v_{k+1}\right), S_{D}\right)=\left\|\left[\begin{array}{c}
M\left(v_{k+1}\right) \\
C\left(v_{k+1}\right)
\end{array}\right] \Phi_{v_{k}}^{v_{k+1}} P_{u} u\right\|, u^{T} u \leq 1
$$

The distance to $S_{D}$ depends only on the instants $v_{k}$ and $v_{k+1}$ and on the amount of navigation uncertainty considered. If the uncertainty is high then a smaller interval between the impulses might be necessary to ensure the stability of the system. The robustness of the proposed control method is validated through numerical tests in the following section.

\section{NUMERICAL ANALYSIS}

The performances of the control method (30) are analysed through realistic closed-loop simulations. The same philosophy as for MPC control [13] is used to implement the closed loop: at each time step a pair of two impulses is calculated but only the first one is applied.

The relative motion is propagated using the Gauss Variational Equations. The sensor noise is modelled by adding an uncorrelated white noise on the relative position and velocity respectively. The reference orbit has a semi-major axis of $7011 \mathrm{~km}$ and an eccentricity that depends on the simulation scenario. For each eccentricity a reference periodic trajectory specified through the vector of parameters $D$ is obtained using the algorithm in [12]. All the reference trajectories are required to evolve inside a tolerance box $X_{t o l}=[50 ; 25 ; 25][\mathrm{m}]$ around the final position $X_{f}=\left[\begin{array}{lll}100 & 0 & 0\end{array}\right]$ $[\mathrm{m}]$. The dimensions of the tolerance box have been chosen to guarantee feasible periodic trajectories for a wide range of eccentricities.

The ability of the method to accommodate different types of reference trajectories is analysed next, along with the influence that the interval between controls and the navigation uncertainties have on its performances.

\section{A. Influence of the eccentricity}

The influence of the eccentricity on the stability and the performance of the control method is analysed by considering different eccentricities for the leader's orbit and different corresponding periodic reference trajectories. The control is applied every $T_{s}=100 \mathrm{~s}$ for simulations lasting over 10 orbital periods. A typical navigation uncertainty of $0.02 \mathrm{~m}$ for the relative position and of $0.002 \mathrm{~m} / \mathrm{s}$ for the relative velocity is considered [8].

A discrete LQ method [14] is used for comparison, since it requires the same amount of calculations. The gain matrix are chosen $Q=I_{6}$ and $R=0_{3}$ since for the two-impulse method no constraints are imposed on the control.

Figure 3 shows the trajectories obtained in simulation, using the two-impulse control method, for each eccentricity,
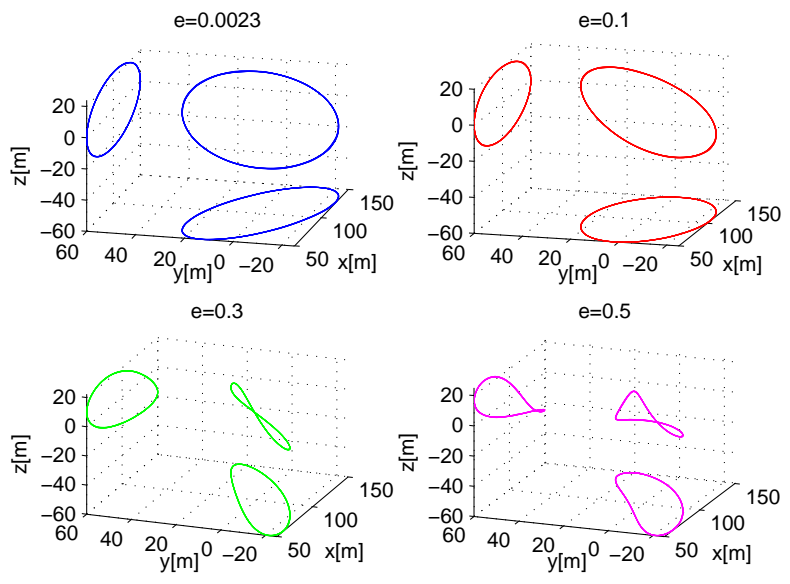

Fig. 3. The simulation trajectories for each eccentricity 
TABLE I

INFLUENCE OF THE ECCENTRICITY

\begin{tabular}{c|c|c|c|c} 
Method & $e$ & $P_{\text {err }}[\mathrm{m}]$ & $V_{\text {err }}\left[\frac{\mathrm{m}}{\mathrm{s}}\right]$ & $\Delta V\left[\frac{\mathrm{m}}{\mathrm{s}} /\right.$ orbit $]$ \\
\hline & 0.0238 & 0.2678 & 0.004 & 0.2554 \\
& 0.1 & 0.2695 & 0.004 & 0.2554 \\
$2 \Delta V$ & 0.3 & 0.3842 & 0.011 & 0.2577 \\
& 0.5 & 0.4986 & 0.037 & 0.2761 \\
\hline \multirow{3}{*}{ LQ } & 0.0238 & 1.6293 & 0.0037 & 0.2002 \\
& 0.1 & 1.5453 & 0.0041 & 0.2002 \\
& 0.3 & 1.8075 & 0.0114 & 0.2005 \\
& 0.5 & 2.9523 & 0.0389 & 0.2099
\end{tabular}

along with their projections on the $x y, x z$ planes. Even if the trajectories become more twisted as eccentricity increases, our method ensures good tracking performances.

Table I gives the maximum of the absolute value of the position error on any axis gives $P_{e r r}$, the maximum of the absolute value of the velocity error on any axis $V_{e r r}$ and the fuel consumption per orbit for each scenario and for each control method (LQ and two-impulse $2 \Delta V$ ). At first sight the two-impulse method shows slightly higher fuel consumption than the LQ method but this is just the price to pay for better tracking performances. Table II shows that in order to reach the same tracking precision of approximately 2 $\mathrm{m}$, our method allows a bigger interval between impulses $T_{S}$ and issues a lower fuel consumption per orbit $\Delta V$. For instance, for an eccentricity $e=0.3$, the same performances are obtained using a control interval 4 times bigger and with 3 times less fuel per orbit.

For higher eccentricities like $e=0.8$ the LQ method does not ensure the stability and the spacecraft drift rapidly from the reference trajectory. The $2 \Delta V$ method has some problems to follow the quickly changing parts of the reference trajectory and shows a growing fuel consumption over the 10 orbital periods considered. This suggests an unstable behaviour that may be explained by the fact that for very eccentric orbits a smaller time step is needed for the control. It could also come from the fact that the orbital dynamics used to build the reference trajectory are no longer valid for such high eccentricities.

\section{B. Influence of the interval between controls}

The frequency of control execution is a key parameter both from fuel consumption and stability point of view. For each eccentricity, several values for the interval between consecutive impulses are used to analyse the fuel consumption per orbit and the maximum distance to the invariant set $S_{D}$

TABLE II

FUEL CONSUMPTION FOR SAME TRACKING PRECISION $P_{\text {err }}=2 \mathrm{~m}$

\begin{tabular}{c|c|c|c|c} 
& \multicolumn{2}{|c|}{$2 \Delta V$} & \multicolumn{2}{c}{$\mathrm{LQ}$} \\
\hline$e$ & $T_{s}[\mathrm{~s}][\mathrm{m}]$ & $\Delta V\left[\frac{m}{s} /\right.$ orbit $]$ & $T_{s}[\mathrm{~s}][\mathrm{m}]$ & $\Delta V\left[\frac{m}{s} /\right.$ orbit $]$ \\
\hline 0.0238 & 600 & 0.0411 & 130 & 0.1533 \\
0.1 & 600 & 0.0402 & 130 & 0.1537 \\
0.3 & 400 & 0.0682 & 100 & 0.2005 \\
0.5 & 250 & 0.1383 & 50 & 0.4036
\end{tabular}

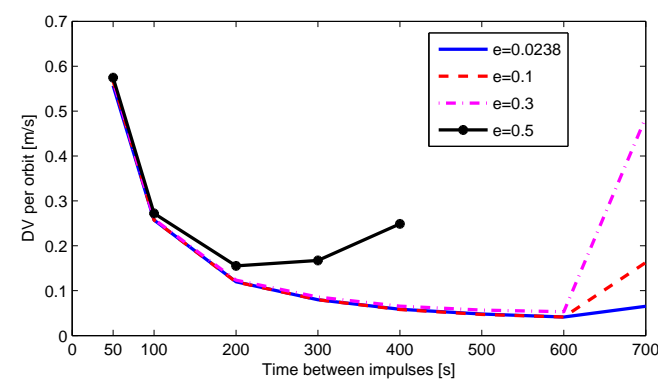

Fig. 4. Influence of the interval between controls on the fuel consumption

(computed with a 2-norm in (26)). A navigation uncertainty of $0.02 \mathrm{~m}$ on the relative position and of $0.002 \mathrm{~m} / \mathrm{s}$ on the relative velocity is considered for simulations running over 30 orbital periods.

Figure 4 shows that, before reaching a certain critical value, the larger the interval between controls the smaller the fuel consumption. After this value the fuel consumption quickly increases, as the system approaches the stability limit. The critical control interval depends on the orbit's eccentricity as highly eccentric orbits require a smaller control step.

Figure 5 shows that there is a direct link between the interval between controls, the fuel consumption and the maximum distance to the invariant set $S_{D}$. A large distance to the invariant set is equivalent to high fuel consumption, large tracking errors and/or instability. Considering a small control interval does not necessarily imply a smaller distance to $S_{D}$ since the system could be moved away from the reference trajectory by unnecessary thrusting caused by the navigation errors.

\section{The influence of navigation uncertainty}

The influence of the navigation uncertainty on the tracking precision and on the fuel consumption is analysed next. For an eccentricity of 0.3 , several values for the control interval $T_{S}$ and for the measurement noise $\delta P, \delta V$ are considered.

Figure 6 shows that when there is no measurement noise, the choice of the control interval does not impact significantly the fuel consumption. As the measurement uncertainty gets higher it becomes more fuel efficient to consider a large interval between consecutive corrections in order to avoid unnecessary thrusting due to inaccurate measures.

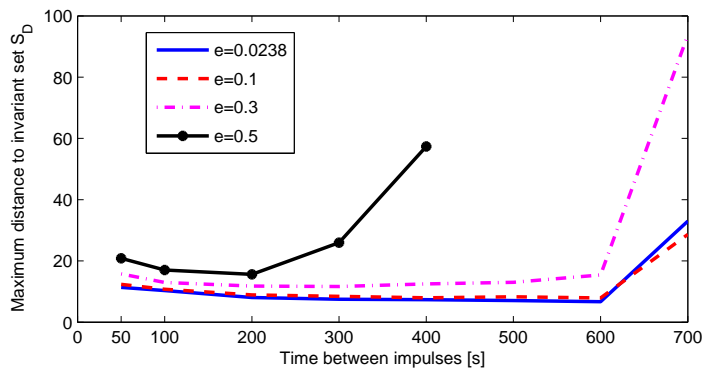

Fig. 5. Influence of the interval between controls on the distance to $S_{D}$ 


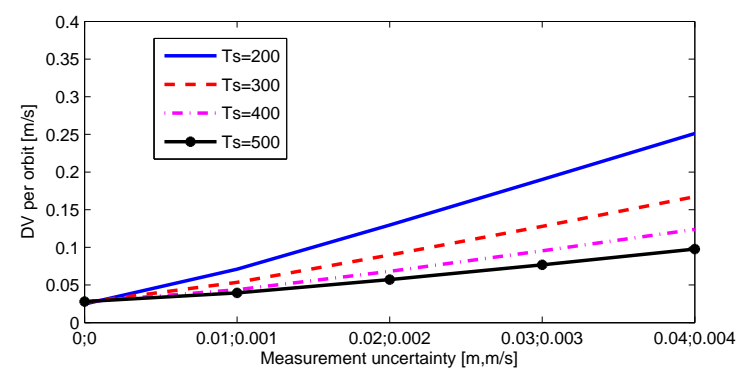

Fig. 6. Influence of the measurement noise on the fuel consumption

Table III gives, for $T_{s}=500 \mathrm{~s}$, the absolute value of the maximum position error $P_{e r r}$ and of the maximum velocity error $V_{e r r}$, along with the fuel consumption per orbit $\Delta V$ and the maximum distance to the invariant set $S_{D}, d_{\max }$. It shows that even for high navigation uncertainties the tracking performances maintain acceptable bounds and that the distance to the invariant set increases with the measurement noise.

\section{Conservativeness of the fixed reference trajectory}

For some types of missions, the relative trajectory itself might not be very important as long as it is periodic and it evolves in a specified region. Orbital perturbations could bring the spacecraft to a configuration where less fuel is required for the transfer to a different neighbouring periodic relative trajectory, which evolves inside the specified region, than for steering back to the current one.

To address this issue, the analytical two-impulse strategy is compared with the semi-definite program we presented in [12] where the number of impulses is equal to 2 (called here 2SDP). An orbit with $e=0.3$, a re-plan time of $T_{S}=100$ $\mathrm{s}$ and a navigation uncertainty of $0.02 \mathrm{~m}$ for position and $0.002 \mathrm{~m} / \mathrm{s}$ for velocity are considered for the comparison of the two methods.

Figure 7 shows the obtained relative trajectories, along with their $x y$ and $x z$ projections. For the 2SDP method the trajectory is free to evolve inside the specified tolerance box so it changes dimensions and it changes orientation. This freedom is translated into a fuel consumption per orbit that is 10 times smaller than the two-impulse strategy.

\section{CONCLUSIONS}

An analytical method for computing a two-impulse control law that stabilizes the relative motion with respect to the invariant set composed of the states belonging to a desired periodic trajectory is presented. The evaluation of

TABLE III

INFLUENCE OF THE NAVIGATION UNCERTAINTY

\begin{tabular}{c|c|c|c|c}
$\delta P[\mathrm{~m}], \delta V\left[\frac{m}{s}\right]$ & $\Delta V\left[\frac{m}{s} /\right.$ orbit $]$ & $P_{\text {err }}[\mathrm{m}]$ & $V_{\text {err }}\left[\frac{m}{s}\right]$ & $d_{\max }$ \\
\hline 0,0 & 0.0280 & 1.7529 & 0.0079 & 7.6698 \\
$0.01,0.001$ & 0.0394 & 1.8834 & 0.0081 & 9.0410 \\
$0.02,0.002$ & 0.0572 & 2.1505 & 0.0103 & 11.2018 \\
$0.03,0.003$ & 0.0768 & 2.5151 & 0.0125 & 13.4797 \\
$0.04,0.004$ & 0.0976 & 2.8798 & 0.0148 & 17.3393
\end{tabular}

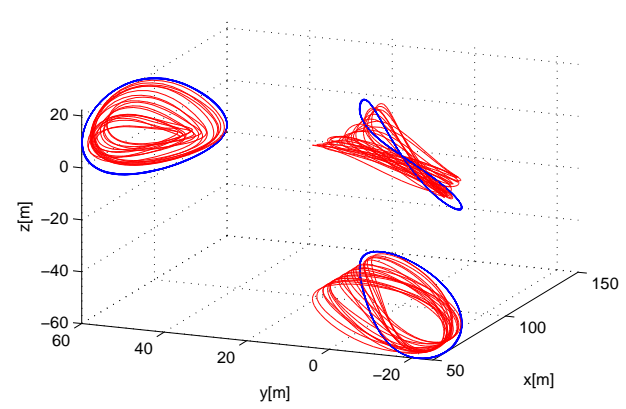

Fig. 7. Comparison between 2DV and 2SDP trajectories

the maximum distance to the invariant set can be used as a criterion for choosing the best frequency of control execution in the presence of navigation uncertainty. With very few computational effort, the method provides good tracking performances for a wide range of reference trajectories and appears to be quite robust to typical levels of measurement noise. However, on-line optimization of the reference trajectory can help reducing the fuel consumption.

\section{REFERENCES}

[1] J. H. Saleh, D. E. Hastings, E. S. Lamassoure, and D. J. Newman, "Flexibility and the Value of On-Orbit Servicing: New CustomerCentric Perspective," Journal of Spacecraft and Rockets, vol. 40, no. 2, pp. 279-291, Mar. 2003.

[2] G. Inalhan, J. How, and M. Tillerson, "Relative Dynamics and Control of Spacecraft Formations in Eccentric Orbits," Journal of Guidance, Control, and Dynamics, vol. 25, no. 1, pp. 48-59, Jan. 2002.

[3] P. Sengupta and S. R. Vadali, "Relative Motion and the Geometry of Formations in Keplerian Elliptic Orbits," Journal of Guidance, Control, and Dynamics, vol. 30, no. 4, pp. 953-964, 2007.

[4] L. S. Breger, G. Inalhan, M. Tillerson, and J. How, "Cooperative Spacecraft Formation Flying: Model Predictive Control with Openand Closed-Loop Robustness," in Modern Astrodynamics, P. Gurfil, Ed. Butterworth-Heinemann, 2007, ch. 8, pp. 237-277.

[5] J.-F. Hamel and J. de Lafontaine, "Autonomous guidance and control of Earth-orbiting formation flying spacecraft: Closing the loop," Acta Astronautica, vol. 63, no. 11-12, pp. 1246-1258, Dec. 2008.

[6] J. D. Biggs and C. McInnes, "Time-Delayed Feedback Control in Astrodynamics," Journal of Guidance, Control, and Dynamics, vol. 32, no. 6, pp. 1804-1811, 2009.

[7] M. Bando and A. Ichikawa, "Satellite Formation and Reconfiguration with Restricted Control Interval," Journal of Guidance, Control, and Dynamics, vol. 33, no. 2, pp. 607-615, Mar. 2010.

[8] M. Tillerson, G. Inalhan, and J. How, "Co-ordination and control of distributed spacecraft systems using convex optimization techniques," International Journal of Robust and Nonlinear Control, vol. 12, no. 2-3, pp. 207-242, Feb. 2002.

[9] K. Yamanaka and F. Ankersen, "New state transition matrix for relative motion on an arbitrary elliptical orbit," Journal of guidance, control, and dynamics, vol. 25, no. 1, pp. 60-66, 2002.

[10] J. Tschauner, "Elliptic orbit rendezvous," AIAA Journal, vol. 5, no. 6 , pp. 1110-1113, 1967.

[11] H. K. Khalil, Nonlinear Systems Third Edition. New Jersey: Pearson Education International, 2000.

[12] G. Deaconu, C. Louembet, and A. Théron, "Constrained periodic spacecraft relative motion using non negative polynomials," in American Control Conference (ACC), 2012.

[13] J. Maciejowski, Predictive Control with Constraints. Harlow: Pearson Education, 2002

[14] D. E. Kirk, Optimal Control Theory An Introduction. Dover Publications, Inc., 2004. 